\title{
Amiodarone-Induced Thyroid Storm Causing Sustained Monomorphic Ventricular Tachycardia Treated With Plasmapheresis: A Challenging Clinical Case
}

\author{
Vandan D. Upadhyaya ${ }^{a}$, Steven Douedi ${ }^{a}$, d , Monika Akula ${ }^{b}$, Krishna K. Chalasani ${ }^{b}$, \\ Matthew D. Sayboltc, Mohammad Hossain ${ }^{\mathrm{a}}$
}

\begin{abstract}
Thyroid storm is a rare endocrine emergency with a high mortality rate approaching $20 \%$. It manifests with the exaggerated symptoms of hyperthyroidism including hyperpyrexia, hypotension, cardiac arrhythmias, and death. The mainstay of treatment includes supportive intensive care and initiation of thionamides, beta blockers, corticosteroids, and if necessary anti-arrhythmics in the presence of refractory ventricular arrhythmias. We describe a case of amiodarone-induced thyrotoxicosis and thyroid storm leading to refractory ventricular tachycardias treated with plasmapheresis when anti-arrhythmic therapy became contraindicated. Amiodarone-induced thyroid storm can lead to monomorphic ventricular tachycardia. Under the circumstances of limited medical therapies, plasmapheresis can be an effective treatment option.
\end{abstract}

Keywords: Amiodarone; Thyrotoxicosis; Ventricular tachycardia; Plasmapheresis; Thyroid storm

\section{Introduction}

Thyroid storm is a rare endocrine emergency that presents with exaggerated clinical manifestations of thyrotoxicosis. Based on the National Inpatient Sample database, a retrospective longitudinal analysis found one in six patients with a discharge diagnosis of thyrotoxicosis was also diagnosed with thyroid storm [1]. Thyrotoxicosis associated with thyroid storm carries with it a 12-fold higher mortality rate than thyrotoxicosis

Manuscript submitted March 4, 2020, accepted March 16, 2020

aDepartment of Medicine, Hackensack Meridian Health Jersey Shore University Medical Center, Neptune, NJ 07753, USA

bDepartment of Endocrinology, Hackensack Meridian Health Jersey Shore University Medical Center, Neptune, NJ 07753, USA

'Department of Cardiology, Hackensack Meridian Health Jersey Shore University Medical Center, Neptune, NJ 07753, USA

${ }^{\mathrm{d} C}$ Corresponding Author: Steven Douedi, Department of Medicine, Hackensack Meridian Health Jersey Shore University Medical Center, Neptune, NJ 07753, USA. Email: Steven.Douedi@hackensackmeridian.org

doi: https://doi.org/10.14740/jmc3450 alone [1]. Thyroid storm traditionally has been identified as a clinical syndrome involving thyrotoxicosis, altered mentation, hyperthermia, and a precipitating event [2]. Currently, there are no universally accepted clinical criteria for the diagnosis of thyroid storm. Proposed in 1993, the Burch-Wartofsky score is a scoring metric based on clinical findings used for the identification of thyroid storm [3]. Current treatment modalities revolve around decreasing hormone synthesis, inhibiting the release of thyroid hormone, and blocking peripheral effects of existing systemic thyroid hormone [4].

Amiodarone, a type III antiarrhythmic approved for the use of ventricular and supraventricular arrhythmias, is $37 \%$ iodine by molecular weight. Side effects are many, including thyroid dysfunction, both hyper- and hypothyroidism [5]. Amiodarone-induced thyrotoxicosis (AIT) results either from increased synthesis of T4 and T3 (AIT type I), or from the destructive thyroiditis resulting in excess hormone release (AIT type II) [6]. In a retrospective analysis, incidence of AIT was found to be associated with a 2.7-fold increased risk of major adverse cardiovascular events including myocardial infarction, stroke, heart failure, or ventricular arrhythmias compared to euthyroid patients on amiodarone [7].

We report a case and management of AIT resulting in severe thyroid storm and monomorphic ventricular tachycardia.

\section{Case Report}

A 75-year-old man with a history of ischemic cardiomyopathy status post coronary artery bypass graft (CABG), chronic atrial fibrillation on amiodarone, heart failure with reduced ejection fraction (EF) of $25-30 \%$, primary prevention implantable cardiac defibrillator (ICD), and non-toxic large left-sided colloid thyroid nodule presented to the emergency department after an episode of syncope after a prodrome of feeling faint while walking. On presentation he had anterograde amnesia following the event but was alert and not altered. He was afebrile and normotensive but tachycardic with heart rate (HR) 140 - 160 beats per minute (bpm). On physical exam he appeared anxious and tachypneic. Neck exam revealed a non-tender 6-cm thyroid nodule and cardiac auscultation revealed I/VI holosystolic murmur. His home medications included amiodarone and digoxin for underlying chronic atrial fibrillation started 4 months prior, 
Table 1. Highlights TSH and Free Thyroxine Levels (Free T4) and Laboratory Findings Available at the Time of Admission

\begin{tabular}{lll} 
& Date of admission & Two years prior \\
\hline TSH & $<0.01$ & 0.652 \\
Free T4 & 5.46 & 0.65 \\
\hline
\end{tabular}

TSH: thyroid-stimulating hormone.

metoprolol, dabigatran, and rosuvastatin. Initial electrocardiogram (EKG) demonstrated monomorphic ventricular tachycardia and ICD interrogation reveled that he had been defibrillated eight times during his syncopal episode. During the interview period he continued to receive tachytherapies from his implanted device including three defibrillations. He was treated with intravenous (IV) amiodarone, lidocaine, and ultimately intubated and sedated for unremitting ventricular tachycardia storm.

In the intensive care unit, workup yielded undetectable thyroid-stimulating hormone (TSH) and an elevated free thyroxine of $5.46 \mathrm{ng} / \mathrm{dL}$. Thyroid function tests prior to initiation of amiodarone were reviewed and previously were within normal limits (Table 1). The patient's Burch-Wartofsky score was calculated to be 60. Diagnosis of severe thyrotoxicosis with thyroid storm was suspected and the patient was initiated on steroids, cholestyramine, potassium iodide, and esmolol. He was unable to be started on thionamides and lithium due to acute liver and renal failure. Continued workup demonstrated elevated thyroid stimulating immunoglobin M (IgM) antibodies and thyrotropin receptor antibodies while thyroid peroxidase antibodies were negative.

After emergency multi-disciplinary evaluation, his acute thyroid storm was determined to be due to AIT, a diagnosis of exclusion. As a result, amiodarone was ceased. The patient continued to deteriorate and experienced acute decompensated heart failure. Echocardiogram exhibited severe global hypokinesis and an EF of $11 \%$. Plasmapheresis was initiated; however, his condition continued to deteriorate and he developed refractory shock and ultimately expired.

\section{Discussion}

Ventricular tachycardia induced by thyroid storm was first reported by Jao et al in 2004 [8]. However, the patient described in that case had a 7-year history of hyperthyroidism with irregular intake of medications. In the case we described above, the patient has no known history of thyroid disease other than a non-toxic thyroid nodule. Treatment of hemodynamically stable ventricular arrythmias includes IV antiarrhythmic medications such as amiodarone or beta-blocking agents. We believe that chronic amiodarone treatment precipitated our patient's thyroid storm and thus it was not continued and treatment for thyrotoxicosis was initiated. However due to acute liver failure and hypotension, thionamides and beta blockers were also contraindicated. Ultimately, we offered plasmapheresis as an alternative therapy. Simsir et al previously reported 46 patients in thyroid storm and the efficacy of plasmapheresis in decreasing free T3 and T4 levels [9]. Muller et al determined that plasmapheresis is capable of clearing amiodarone and its metabolites which are highly bound to plasma proteins, as well as free T3 and T4 molecules by up to $20-60 \%$ [10]. Cases have been reported where therapeutic plasma exchange (TPE) was initiated for type 2 amiodarone-induced hyperthyroidism [10]. Given the dual reported benefits and our patient's poor candidacy for standard of care, plasmapheresis was offered. Thus, when medical management of thyroid storm is limited, plasmapheresis may be an effective alternative option.

\section{Conclusions}

Thyroid storm is a rare endocrine emergency that carries a high mortality and is challenging to treat. Mainstay therapy requires patients to receive agents to decrease hormone synthesis, inhibit hormone release, and block action of existing systemic thyroid hormone. Amiodarone-induced thyroid storm poses its own challenges compounding on an otherwise critical presentation. We pose a case of amiodarone-induced thyroid storm presenting with monomorphic sustained ventricular tachycardia that was treated with plasmapheresis highlighting an alternative, and in this case, unsuccessful therapy in the treatment of this severe condition.

\section{Acknowledgments}

None to declare.

\section{Financial Disclosure}

None to declare.

\section{Conflict of Interest}

None to declare.

\section{Informed Consent}

The patient described in the case report had given informed consent for the case report to be published.

\section{Author Contributions}

Each author has individually been involved in and has made substantial contributions to case selection, case presentation, discussion, drafting manuscript and revision and final approval.

\section{Data Availability}

All data and information regarding this manuscript are present within the text. 


\section{References}

1. Galindo RJ, Hurtado CR, Pasquel FJ, Garcia Tome R, Peng L, Umpierrez GE. National trends in incidence, mortality, and clinical outcomes of patients hospitalized for thyrotoxicosis with and without thyroid storm in the United States, 2004-2013. Thyroid. 2019;29(1):36-43.

2. Angell TE, Lechner MG, Nguyen CT, Salvato VL, Nicoloff JT, LoPresti JS. Clinical features and hospital outcomes in thyroid storm: a retrospective cohort study. J Clin Endocrinol Metab. 2015;100(2):451-459.

3. Burch HB, Wartofsky L. Life-threatening thyrotoxicosis. Thyroid storm. Endocrinol Metab Clin North Am. 1993;22(2):263-277.

4. Nayak B, Burman K. Thyrotoxicosis and thyroid storm. Endocrinol Metab Clin North Am. 2006;35(4):663-686, vii.

5. Martino E, Bartalena L, Bogazzi F, Braverman LE.
The effects of amiodarone on the thyroid. Endocr Rev. 2001;22(2):240-254.

6. Bartalena L, Brogioni S, Grasso L, Bogazzi F, Burelli A, Martino E. Treatment of amiodarone-induced thyrotoxicosis, a difficult challenge: results of a prospective study. J Clin Endocrinol Metab. 1996;81(8):2930-2933.

7. Yiu KH, Jim MH, Siu CW, Lee CH, Yuen M, Mok M, Shea YF, et al. Amiodarone-induced thyrotoxicosis is a predictor of adverse cardiovascular outcome. J Clin Endocrinol Metab. 2009;94(1):109-114.

8. Jao YT, Chen Y, Lee WH, Tai FT. Thyroid storm and ventricular tachycardia. South Med J. 2004;97(6):604-607.

9. Simsir IY, Ozdemir M, Duman S, Erdogan M, Donmez A, Ozgen AG. Therapeutic plasmapheresis in thyrotoxic patients. Endocrine. 2018;62(1):144-148.

10. Muller C, Perrin P, Faller B, Richter S, Chantrel F. Role of plasma exchange in the thyroid storm. Ther Apher Dial. 2011;15(6):522-531. 